\title{
A New Approach for Sub-Channels Assignment in Downlink Fourth Generation Mobile Systems
}

\author{
${ }^{1,2}$ Fakhreddine Khelifa and ${ }^{2}$ Abdelaziz Samet \\ ${ }^{1}$ Research Department, Studies and Research Telecommunications Center, \\ Ariana, 2088, \\ ${ }^{2}$ Research Units: Electronics Systems and Components, \\ Polytechnic School of Tunisia, EPT, University of Carthage, Tunis, 2078, Tunisia
}

\begin{abstract}
Problem statement: We proposed a new heuristic method for subchannels allocation problem in the context. Approach: In this system, OFDM transmission method has been used, in order to reduce the effect of multipath fading, OFDMA to achieve multiple access schemes and flexibility in resource allocation. Results: An adaptive method for subchannels allocation is necessary in order to exploit the multiuser diversity, to respect real time constraints and to maximize the capacity system. The proposed algorithm is based in the Channel State Information (CSI) of total mobiles stations present in the cell. The idea of this method is based in the statistic parameters of the gain of frequency response channel for every mobile station. These parameters are the key to organize the different MS's and subchannels priority. Conclusion/Recommendations: Numerical results showed that the proposed algorithm outperforms an allocation scheme in terms of Bloc Error Rate, Higher system capacity and Fairness among active users, compared to others previous methods.
\end{abstract}

Key words: Multiuser diversity, subchannel allocation, dynamic resource allocation, fast fading, Channel State Information (CSI), Long Term Evolution (LTE), transmit power, rate adaptive, numerical results

\section{INTRODUCTION}

A long way in a remarkably short time has been travelled in the history of wireless mobile communication. Looking past, wireless access technologies have experienced several evolutions aimed at unified target: performance and efficiency in high mobile data rate and large bandwidth according to the Quality Of Services (QOS) required by users. To fulfill such target, new challenges have been imposed for wider band high data rate technologies such as Long Term Evolution (LTE) and Mobile Worldwide interoperability for Microwave Access (WiMAX), referred as IEEE 802.16e standard. The main objective of 4 th generation $(4 \mathrm{G})$ technologies is to support a large number of user randomly located in the cell with flexible QOS requirement. OFDMA, also referred as Multiuser Orthogonal Frequency Division Multiplexing (OFDM), is considered as a key step in the evolution of packet access wireless networks, due to its capability to mitigate Inter-Symbol-Interference (ISI), tolerance to multipath propagation and frequency selective fading.
In OFDMA based systems, the available spectrum is divided into overlapping frequency subcarriers which leads to efficient use of the available spectrum. By exploiting the multiuser diversity, OFDMA allocates different subcarriers to different users based on their Channel State Information (CSI) (Zunxiong et al., 2009).

In order to reduce the signaling overhead, recent researches have defined a Resource Unit (RU) that consists of a set of subcarriers. It is referred in LTE as Resource Block (RB) and subchannel in $802.16 \mathrm{~m}$ standard (Sen et al., 2009). In the literature, two methods for RU construction are proposed: "distributed" mode where scattered subcarriers over the whole bandwidth are chosen to build the RU and "adjacent" mode where a group of consecutive subcarriers denotes the RU. In the context of mobility and high mobility, most popular literatures recommend to use distributed method in this case (Sen et al., 2009) rather than adjacent method because of its difficulty to affect the best subchannels to users dynamically. Ideally, is to adapt the contiguous method in context of mobility and high mobility in order to 
optimize the resource utility and to maximize the total capacity of the system.

In recent years, many dynamic resource allocation schemes for the OFDMA downlink systems have been developed to close the optimal solution of optimizing problems that aim to achieve the highest throughput with the minimum transmit power either with the users' data rates as the constraint and the total transmit power as the objective function or with the constraint on the power and the total throughput of the system as the objective, referred respectively as Margin Adaptive (MA) (Cheong et al., 1999; Liejune et al., 2011; Yu, and Lui, 2006; Seong et al., 2006) and Rate Adaptive (RA) (Jang and Lee, 2003) optimization problems. There is also a third type that aims to achieve the highest possible throughput by maintaining fairness among users (Rhee and Cioffi, 2000; Anas et al., 20011; Marabissi et al., 2008; Papoutsis et al., 2010).

\section{METERIALS AND METHODS}

In this study, we propose an adaptive resource allocation algorithm in downlink OFDMA systems. Our main objective is to maximize the total system capacity, while maintaining fairness among users.

The remainder of this study is described as follows. In Section 2, related works are studied. In Section 3 and 4 the optimization problem and system model are formulated. Then, the novel adaptive resource allocation algorithm and the computational complexity are described, respectively, in details in Section 5 and 6. In Section 7, numerical results and performance analysis are provided. Finally, we conclude this presented work in section 8 .

Related works: In the literature, MA problems have been less investigated than the RA problems. They aim to minimize the transmit power with the users' data rates as the constraint. In (Cheong et al., 1999), the authors attempted to minimize the total transmit power under a fixed performance requirement and a given set of user data rates. In the formulation of transmit power minimization problem, authors in (Cheong et al., 1999) do not allow more than one user to share a subcarrier. To find efficient suboptimal algorithms, many works have considered convex relaxation methods by introducing time-sharing or frequency-sharing variables (Rhee and Cioffi, 2000). However, this approach may introduce significant loss in optimality because of its relaxation of the data rate constraints and its high complexity in implementation. To cope with these inconveniences, authors in (Yu and Lui, 2006) make progress toward numerical solution of non-convex optimization problems for multicarrier systems based on the Lagrangian dual of these non-convex problems. Motivated by results proved in (Yu and Lui, 2006), authors Seong et al. (2006) resolve OFDMA downlink resource allocation problems in the dual domain by using Lagrange dual decomposition.

Recently, several algorithms have been proposed to resolve RA problems. They aim to maximize the total data rate of the system with power constraint. In (Jang and Lee, 2003), it is proved that the sum of the users' data rates is maximized when each subcarrier is assigned to the user with the best subcarrier gain. In this study, the problem is divided into two steps: subcarrier assignment and power allocation. By the two step approach, the data rate maximization problem is simplified and may be solved analytically. Authors in (Jang and Lee, 2003) restrict their focus on exclusive assignment of each subcarrier to only one user with the best channel gain. In the second step, they determine the amount of transmit power to be allocated on subcarriers by using a water-filling algorithm and a standard Lagrange multiplier technique in order to determine the water level.

In the literature, several researches resolve RA problems by using water-filling algorithm in the power allocation step because of its capability to perform an optimal solution. However, this is true only in the case of single user systems or multi-users systems with a fixed resource assignment for example in FDMA and TDMA with non-adaptive fixed resource allocation, but, the maximized rate reached by the static resource allocation algorithms using water-filling algorithm is far below the rate that can be achieved by adaptive resource allocation schemes. Furthermore, the water-filling approach seems to be not a powerful tool in $(4 \mathrm{G})$ wireless networks that are characterized by multi-services, mobility and channel time variations. Such wireless networks require instantaneous channel estimations and feedbacks that cannot be performed by water-filling algorithm due to its high computational complexity.

In order to reduce the inconvenience of maximum sum capacity algorithm which is the lack of fairness among users, several researches introduce a fairness criterion in order to reduce resource monopolization by set of users experiencing a good channel gain. Hence, users with low channel gain may not receive data due to lack of resources. In the literature, three approaches are introduced to enforce a fairer behavior of the system such as max-min fairness, proportional fairness and hard fairness. In (Rhee and Cioffi, 2000), fairness in the resource allocation is incorporated through maximizing the minimum data rate among users under the total transmit power constraint. However, the main inconvenience of max-min approach rises when users 
with best channel conditions obtain a lower number of resources with respect to others with worse channel conditions. Moreover, in (4G) wireless networks characterized by multi-services requirements and multiuser diversity, the max-min method seems to be not powerful. To avoid this inconvenience, a proportional fairness allocation strategy is proposed. In (Marabissi et al., 2008), the maximum capacity for each user is estimated accordingly to(Rhee and Cioffi, 2000). In this study, the slot allocation for which the capacity values assigned to the users are proportional to the maximum capacity values expressed as:

$$
\mathrm{C}_{0}: \mathrm{C}_{1}: \ldots: \mathrm{C}_{\mathrm{K}-1}=\mathrm{C}_{\max , 0}: \mathrm{C}_{\max , 1}: \ldots: \mathrm{C}_{\max , \mathrm{K}-1}
$$

where, $\mathrm{K}$ is the total number of users. In this case, user $\mathrm{k}$ satisfying $\mathrm{R}_{\mathrm{k}} / \mathrm{C}_{\max , \mathrm{k}} \leq \mathrm{R}_{\mathrm{i}} / \mathrm{C}_{\max , \mathrm{i}}$ is more prior to choose the next available subcarrier. Authors in (Anpalagan et al., 2009) aim to maximize the total system throughput while rate proportionality is maintained among the users according to:

$\mathrm{R}_{0}: \mathrm{R}_{1}: \ldots: \mathrm{R}_{\mathrm{K}}=\alpha_{1}: \alpha_{2}: \ldots: \alpha_{\mathrm{K}}$

where, $\left\{\alpha_{1}: \alpha_{2}: \ldots: \alpha_{\mathrm{K}}\right\}$ is the set of predetermined proportional constraints.

Although, a fairness criterion provides a fairer behavior for OFDMA systems, it decreases the total system throughput. The main challenge in recent researches is to maximize the total system data rate while ensuring fairness among users which is the main objective in this study.

Optimization problem: We note by $\mathrm{C}_{\mathrm{k}, \mathrm{n}}(\mathrm{t})$ the information quantity transmitted by user $\mathrm{k}$ on subchannel $\mathrm{n}$ at subframe $\mathrm{t}$ and $\mathrm{T}$ the simulation time where it is a multiple of subframe duration. We introduce also, $X_{k, n}(t)$ as a binary variable where $X_{k, n}(t)$ if subchannel $\mathrm{n}$ is allocated to user $\mathrm{k}$ at time $\mathrm{t}$ and $\mathrm{x}_{\mathrm{k}, \mathrm{n}}(\mathrm{t})=0$ otherwise.

The main objectives in this study are the maximization of the total system throughput and fairness between users. The optimization resource allocation problem can be formulated as follows:

$$
\begin{aligned}
& \operatorname{Maximize} \sum_{\mathrm{t}=1}^{\mathrm{T}} \sum_{\mathrm{k}=1}^{\mathrm{K}} \sum_{\mathrm{n}=1}^{\mathrm{N}} \mathrm{x}_{\mathrm{k}, \mathrm{n}}(\mathrm{t}) \cdot \mathrm{C}_{\mathrm{k}, \mathrm{n}}(\mathrm{t}) \\
& \text { Maximize } \mathrm{J}_{\text {Fairnessndex }}=\frac{\left(\sum_{\mathrm{k}=1}^{\mathrm{K}} \mathrm{t}_{\mathrm{k}, \text { mean }}\right)^{2}}{\mathrm{~K} \cdot \sum_{\mathrm{k}=1}^{\mathrm{K}}\left(\mathrm{t}_{\mathrm{k}, \text { mean }}\right)^{2}}
\end{aligned}
$$

Subject to:

$$
\begin{aligned}
& \mathrm{C}_{1}: \sum_{\mathrm{k}=1}^{\mathrm{K}} \mathrm{x}_{\mathrm{k}, \mathrm{n}}(\mathrm{t})=1, \forall \mathrm{n} \\
& \mathrm{C}_{2}: \sum_{\mathrm{n}=1}^{\mathrm{N}} \mathrm{x}_{\mathrm{k}, \mathrm{n}}(\mathrm{t}) \leq 1, \forall \mathrm{k} .
\end{aligned}
$$

The $\mathrm{t}_{\mathrm{u} \text {,mean }}$ variable is the mean throughput of user $\mathrm{k}$ in a simulation time. This indicator is given by the following formula, where $t_{k, i}$ is the throughput of user $\mathrm{k}$ in sub-frame duration $\mathrm{i}$ :

$\mathrm{t}_{\mathrm{k}, \text { mean }}=1 / \mathrm{n} \sum_{\mathrm{i}=1}^{\mathrm{n}=\mathrm{T} / \mathrm{T}_{\mathrm{SF}}} \mathrm{t}_{\mathrm{k}, \mathrm{i}} \cdot$

The constraints denoted by $\mathrm{C}_{1}$ and $\mathrm{C}_{2}$ ensure that each subchannel is assigned to only one user at the same time $t$. We suppose that the number of active users $\mathrm{K}$ is greater than the number of available subchannels $\mathrm{N}$, meaning $\mathrm{K} \geq \mathrm{N}$. Then the $\mathrm{BS}$ assigns to each user only one subchannel and a subchannel is allocated to only one user during a time slot which is denoted by constraint $\mathrm{C}_{3}$.

Sysem model: In this study an OFDMA system for 4th generation of wireless networks is considered. The system consists of a single Base Station (BS) that servers $\mathrm{K}$ users randomly distributed in a single cell, where $\mathrm{B}$ is the total bandwidth and $\mathrm{N}$ represents the number of subchannels. A subchannel is composed by a group of $\mathrm{M}$ adjacent subcarriers, with $\mathrm{N}=\mathrm{L} / \mathrm{M}$ and $\mathrm{L}$ is the total number of available subcarriers.

In this study, the available spectrum is divided into 1024 subcarriers and the subchannel is composed by 18 subcarriers contiguous in frequency (16 data subcarriers and 2 pilot subcarriers). We assume that a subchannel may be assigned to only one user where each subchannel is a Rayleigh fast fading channel that has $\mathrm{P}$ paths with an Additive White Gaussian Noise (AWGN). It is assumed also that the Channel State Information (CSI) for each user is well known by the BS. We suppose that the transmitter and the receiver are equipped with a single antenna. We can define the channel gain array $\mathrm{H}$ as follows:

$$
\mathrm{H}=\left(\begin{array}{cccc}
\mathrm{h}_{11} & \mathrm{~h}_{12} & \cdot & \mathrm{h}_{1 \mathrm{~N}} \\
\mathrm{~h}_{21} & \mathrm{~h}_{22} & \cdot & \mathrm{h}_{2 \mathrm{~N}} \\
\cdot & \cdot & \cdot & \cdot \\
\mathrm{h}_{\mathrm{k} 1} & \cdot & \cdot & \mathrm{h}_{\mathrm{KN}}
\end{array}\right)
$$

Where: 
$\mathrm{K}$ and $\mathrm{N}$ = present, respectively, the number of users and the number of available subchannels in the system

$\mathrm{h}_{\mathrm{k}, \mathrm{n}} \quad=$ The channel gain of the $\mathrm{n}^{\text {th }}$ subchannel for to the user $\mathrm{k}$

$\mathrm{A}_{\mathrm{aff}}=\left(\begin{array}{cccc}\alpha_{11} & \alpha_{12} & \cdot & \alpha_{1 \mathrm{~N}} \\ \alpha_{21} & \alpha_{22} & \cdot & \alpha_{2 \mathrm{~N}} \\ \cdot & \cdot & \cdot & \cdot \\ \alpha_{\mathrm{k} 1} & \cdot & \cdot & \alpha_{\mathrm{KN}}\end{array}\right)$

$\mathrm{A}_{\text {aff }}$ denotes the matrix assignment where $\alpha_{\mathrm{kn}}=1$ if the $\mathrm{n}^{\text {th }}$ subchannel is allocated to the user $\mathrm{k}$, otherwise $\alpha_{\mathrm{kn}}=0$. Obviously, $\sum_{\mathrm{n}=1}^{\mathrm{N}} \alpha_{\mathrm{kn}}=1$ when the $\mathrm{n}^{\text {th }}$ subchannel is allocated and $\sum_{n=1}^{N} \alpha_{k n}=0$ otherwise. Thus $\sum_{n=1}^{N} \sum_{k=1}^{K} \alpha_{k n} \leq N$

Considering $\mathrm{h}_{\mathrm{kn}}$ as the frequency response of the $\mathrm{k}^{\text {th }}$ user on $\mathrm{n}^{\text {th }}$ subchannel, it follows the complex Gaussian distribution and its magnitude, called fading factor, follows Rayleigh distribution (Zhu and Wang, 2010). Moreover, we notice that $h_{k n}=\neq h_{k}$ 'n with $k^{\prime} \neq k$.

The Signal to Noise Ratio (SNR) of the $n^{\text {th }}$ subchannel for user $\mathrm{k}$ is expressed as:

$\mathrm{SNR}=10 \log _{10}\left(\frac{\mathrm{P}_{\mathrm{k}}}{\Delta \mathrm{fN}_{0}}\left|\mathrm{~h}_{\mathrm{kn}}\right|^{2}\right)$

where, $\mathrm{P}_{\mathrm{k}}$ and $\Delta \mathrm{f}$ are respectively the transmit power and subcarrier spacing and $\mathrm{N}_{0}$ presents the AWGN noise variance.

In OFDMA-based systems, inter-cell interference occurs at a mobile station, when the BS's of neighboring cells transmits data over a subcarrier used by its serving node. The inter-cell interference phenomenon depends on user location, mobility and frequency reuse factor:

$\operatorname{SINR}_{\mathrm{e}, \mathrm{k}}=10 \log _{10}\left(\frac{\mathrm{P}_{\mathrm{e}}}{\Delta \mathrm{f}\left(\mathrm{I}_{\mathrm{e}, \mathrm{k}}+\mathrm{N}_{0}\right)}\left|\mathrm{h}_{\mathrm{kn}}\right|^{2}\right)$

The downlink quality can be measured by the Signal to Interference plus Noise Ratio (SINR) and expressed as:

The average downlink interference per subchannel $\mathrm{I}_{\mathrm{e}, \mathrm{k}}$ by the $\mathrm{MS}_{\mathrm{k}}$ served by $\mathrm{BS}_{\mathrm{e}}$ is expressed as follows:

$$
I_{e, k}=\sum_{e \neq s} \beta_{e, s} x_{s}\left(\frac{P_{s} G_{s, k}}{L_{s, k}}\right)
$$

Where:
$\mathrm{G}_{\mathrm{s}, \mathrm{k}}=$ Antenna gain

$\mathrm{x}_{\mathrm{s}}=$ Probability that the same subchannel used by the mobile

$\mathrm{k}=$ Used in the same time by another MS served by the $\mathrm{BS}_{\mathrm{s}}$

$\mathrm{L}_{\mathrm{s}, \mathrm{k}}=$ Path loss between $\mathrm{BS}_{\mathrm{s}}$ and the $\mathrm{MS}_{\mathrm{k}}$

Moreover, $\mathrm{P}_{\mathrm{s}}$ denotes the downlink transmit power per subchannel of the $\mathrm{BS}_{\mathrm{s}}$ and $\beta_{\mathrm{s}, \mathrm{k}}$ denotes the interference matrix, where the coefficient $\beta_{\mathrm{s}, \mathrm{k}}$ equals to 1 if cells e and s use the same band and zero otherwise.

The average spectral efficiency of the user $k$ is determined by Adaptive Modulation and Coding (AMC) level according to the estimated SINR is presented in Table 1 and obtained as follows:

$\mathrm{C}_{\mathrm{k}}=\frac{1}{\mathrm{~N}} \sum_{\mathrm{i}=1}^{\mathrm{N}} \alpha_{\mathrm{k}, \mathrm{i}} \mathrm{c}_{\mathrm{i}}$

Adaptive resource allocation: Initialization: Equal power is allocated to groups.

$$
\begin{aligned}
& \Gamma=\{1,2, \ldots, \mathrm{N}\} \\
& \Psi=\{1,2, \ldots, \mathrm{K}\} \\
& \mathrm{h}_{\mathrm{k}, \mathrm{n}}, \forall \mathrm{k} \in \Psi, \forall \mathrm{n} \in \Gamma \\
& \alpha_{\mathrm{k}, \mathrm{n}}=0, \forall \mathrm{k} \in \Psi, \forall \mathrm{n} \in \Gamma \\
& \mathrm{R}_{\mathrm{k}}=0, \forall \mathrm{k} \in \Psi, \forall \mathrm{n} \in \Gamma
\end{aligned}
$$

Users ordering:

For $\mathrm{k}=1$ to $\mathrm{K}$ do

Sort $\mathrm{h}_{\mathrm{k}, \mathrm{n}}$ in descending order

Order user $\mathrm{k}$ according to its best channel gain end for $\mathrm{h}_{\mathrm{k}, \mathrm{j}}$ where $\mathrm{j}=1$

Subchannels allocation:

For $\mathrm{k}=1$ to Kwhere $\operatorname{Order}(\mathrm{k})<\operatorname{order}(\mathrm{k}+1)$

$\mathrm{n} \leftarrow$ find the subchannel $\mathrm{n}$ where the user $\mathrm{k}$ has its best channel gain.

Step (1):

If (unique order $=1$ ) then / only user $\mathrm{k}$ has this

order

\section{Sub-Step (1.a):}

if $\alpha_{1 \leftarrow \mathrm{k}, \mathrm{n}}=0$ then

$\%$ the subchannel $\mathrm{n}$ is not allocated.

$\alpha_{\mathrm{k}, \mathrm{n}} \leftarrow 0$

$\%$ allocate the subchannel $\mathrm{n}$ to user $\mathrm{k}$ $\Gamma=\Gamma-\{\mathrm{n}\}$ Update data rate $R_{k}$ end if

Sub-Step (1.b):

if $\alpha_{1 \leftarrow k, n}=1$ then

$\%$ the subchannel is allocated to more prior user than the actual one $\%$. 
Repeat

$\mathrm{j}=\mathrm{j}+1$

$\{$ Search the next free and best subchannel\}

until $\alpha_{1 \leftarrow k, j}=0$ or $h_{k, j}<h_{k^{*}, j}$

$\left\{\mathrm{k}^{*}\right.$ is the user with less priority than the actual one and $\left.\mathrm{k}^{*} \in[\mathrm{k}+1, \mathrm{~K}]\right\}$.

Jump to sub-step (1.a).

end if

end if

Step (2):

If (uniqueorder $=0$ ) then

\{Two or more users have the same order.\}

Sub-step (2.a)

If (unique subchannel $=0$ ) then

\{Users with the same order do not require the same subchannel.

jump to step (1.a) or sub-step (1.b)

end if

Sub-step (2.b)

If (unique subchannel $=1$ ) then

\{Users with the same order require the same subchannel. $\}$

$$
\text { if } \alpha_{1 \leftarrow \mathrm{k}, \mathrm{n}}=0
$$

$\widehat{\mathrm{k}} \leftarrow$ Determine the user that has the minimum second best subchannel

\{This user has a low chance to get a good subchannel\} Jump to step (1.a).

end if

if $\alpha_{1 \leftarrow \mathrm{k}, \mathrm{n}}=1$ then

$\widehat{\mathrm{k}} \leftarrow$ Determine the user that has the minimum second best subchannel.

end if Jump to sub-step (1.b)

end if

end if

end for

END

\section{RESULT AND DISCUSSION}

Computational complexity: Resource allocation complexity is a main criterion in the design of suboptimal algorithms. In order to analyze the computational complexity of the proposed algorithm, recall that $\mathrm{K}$ refers to the total number of users in the system. $\mathrm{N}$ on the other hand refers to the number of RU's. The initialization step (i) requires constant time for initialization. Then, the RU allocation step sort subchannels in descending order for each user. The sorting process requires $\mathrm{N} \log (\mathrm{N})$ operations for only one user. Sorting subchannels in descending order for all users requires then K.Nlog $(\mathrm{N})$. These operations pertain to the RU allocation and the asymptotic complexity is equal to $\mathrm{O}(\mathrm{K} . \mathrm{N})$.
Numerical results: We present numerical results in order to show the performance of the proposed algorithm compared to (Hwang et al., 2008; Shen et al., 2005). The channel is modeled as a Rayleigh fast fading channel with four paths. The system considered for simulation is the downlink of a single cell that uses 48 subchannels for communications and serves 150 mobile users. We suppose that a subchannel is formed by a group of adjacent subcarriers subdivided in 16 data subcarriers and 2 pilot subcarriers. We have considered a frame duration $\mathrm{TF}=5 \mathrm{~ms}$, a useful symbol time $\mathrm{Ts}=$ $128 \mu$ s and subcarrier spacing $\Delta \mathrm{f}=7.813$.

Table 1 describes Adaptive Modulation Coding (AMC) where each state is represented by a modulation scheme: QPSK, 16QAM and 64QAM and the corresponding coding rate, Forward Error Correction (FEC), according to SINR expressed in decibels (dB).

In order to consider mobility, we assume that the channel state changes every sub-frame delay and the simulation window is equal to 10000 sub-frames Table 2.

The performance of the proposed algorithm is compared with suboptimal existing solutions in terms of total system capacity, fairness, outage probability and Bloc Error Rate (BLER). The performance of the proposed algorithm is compared with those proposed in (Hwang et al., 2008; Shen et al., 2005). The reason for this comparison is as follows. Authors Shen et al. (2005) formulate the problem of maximizing the total system throughput with proportional rate constraints. The principle of this suboptimal subchannel algorithm is to use the subchannel with a high channel-to-noise ratio (SNR) for each user. Authors in (Hwang et al., 2008) propose a heuristic channel allocation and scheduling scheme, where an alternative factor is defined for subchannel allocation. It aims to increase the downlink throughput while maintaining sufficient fairness. Having the same target, suboptimal algorithms proposed in (Hwang et al., 2008; Shen et al., 2005) are then used to evaluate the proposed algorithm performances.

Table 1: Types of modulation and coding

\begin{tabular}{lllll}
\hline Modulation & FEC & SINR $(\mathrm{dB})$ & $\begin{array}{l}\mathrm{c}_{\mathrm{n}} \text { in bit per } \\
\text { symbol }\end{array}$ & Level \\
\hline QPSK & $1 / 3$ & 0.5 & 2 & 1 \\
QPSK & $1 / 2$ & 6.0 & 2 & 2 \\
QPSK & $2 / 3$ & 7.5 & 2 & 3 \\
QPSK & $3 / 4$ & 9.0 & 2 & 4 \\
16-QAM & $1 / 2$ & 12.0 & 4 & 5 \\
16-QAM & $2 / 3$ & 14.5 & 4 & 6 \\
16-QAM & $3 / 4$ & 15.0 & 4 & 7 \\
16-QAM & $5 / 6$ & 17.5 & 4 & 8 \\
64-QAM & $1 / 2$ & 18.0 & 6 & 9 \\
64-QAM & $2 / 3$ & 20.0 & 6 & 10 \\
64-QAM & $3 / 4$ & 21.0 & 6 & 11 \\
64-QAM & $5 / 6$ & 23.0 & 6 & 12 \\
\hline
\end{tabular}




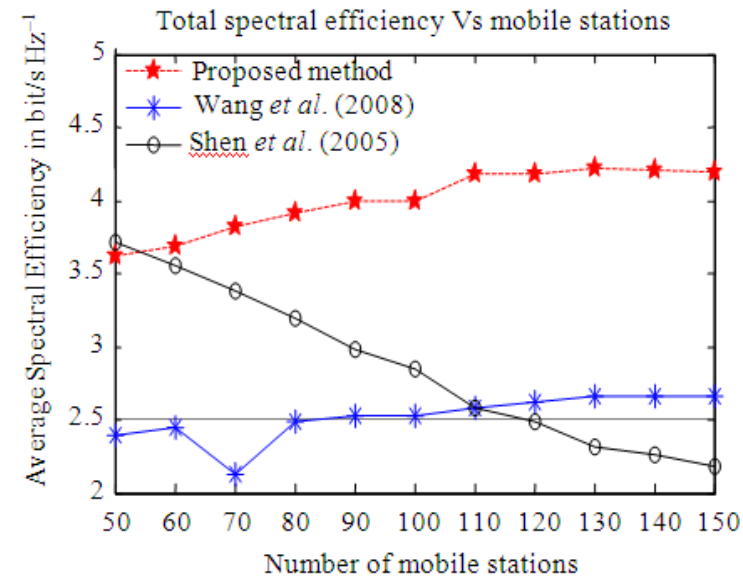

Fig. 1: Total spectral efficiency

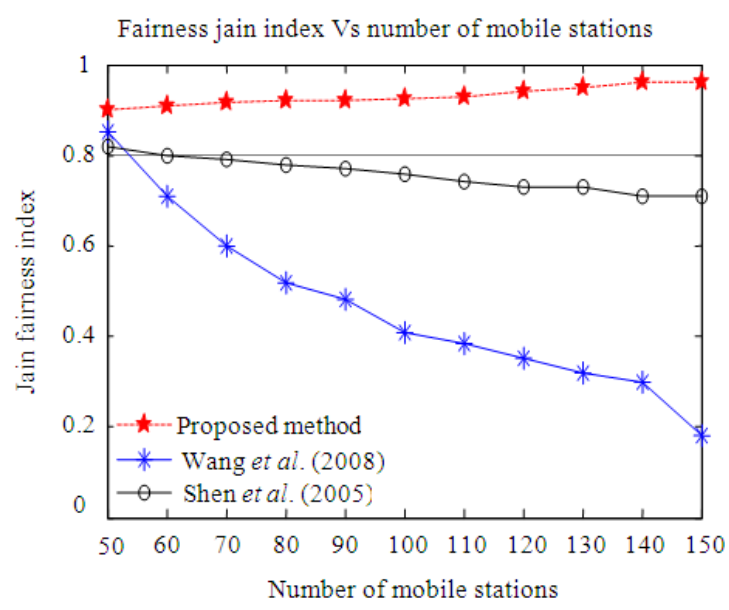

Fig. 2: Jain fairness index

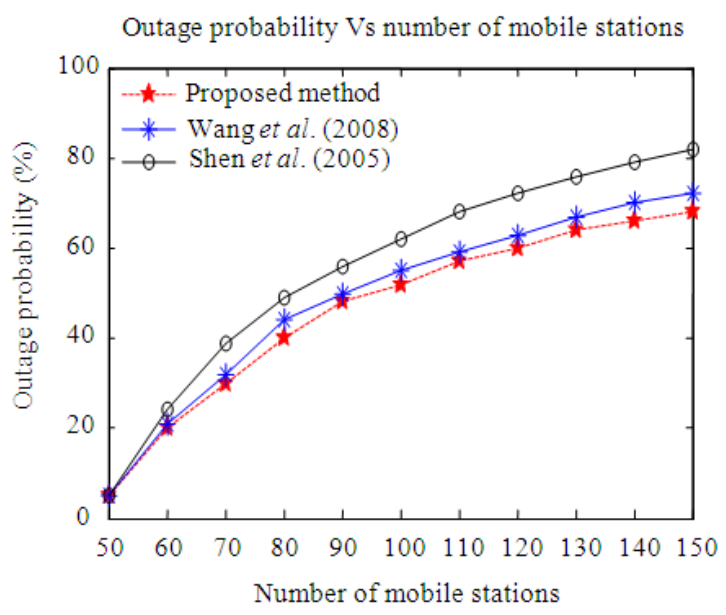

Fig 3: Outage probability

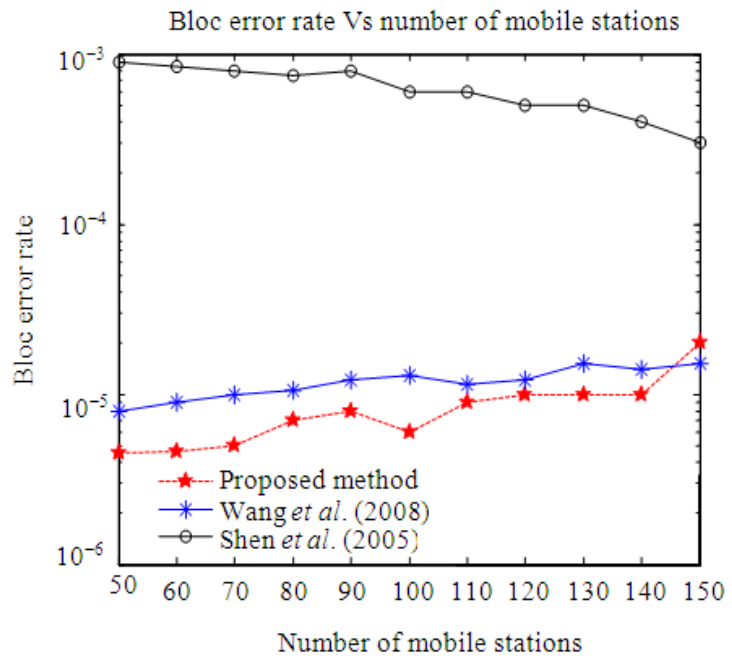

Fig. 4: Bloc error rate

Table 2: OFDMA Parameters for IEEE $802.16 \mathrm{~m}$

\begin{tabular}{lr}
\hline Parameter & Value \\
\hline Super frame delay $(\mathrm{m} \mathrm{sec})$ & 20 \\
Number of frame per super frame & 4 \\
Frame delay (m sec) & 5 \\
Number of sub-frame per frame & 7 \\
Sub-frame delay $(\mu \mathrm{sec})$ & 714,286 \\
\hline
\end{tabular}

Figure 1 compares the average spectral efficiency per subcarrier versus the number of users for different resource allocation schemes. The $\mathrm{X}$ axis represents the number of active users and $\mathrm{Y}$ axis shows the total spectral efficiency expressed in (bit/symbol). This figure shows that the proposed algorithm provides more spectral efficiency than methods proposed in (Hwang et al., 2008; Shen et al., 2005).

To better examine the fairness of these algorithms for different number of users, their performance is shown in Fig. 2. It is obvious that the proposed method provides a fairness index close to 1 Fig. 3 the outage probability versus the number of users. The $\mathrm{X}$ axis represents the number of active users and $\mathrm{Y}$ axis shows the outage probability that represents the rejected users' percentage. This figure shows that the proposed algorithm provides lower outage probability than (Hwang et al., 2008; Shen et al., 2005) methods and then, satisfies greater number of users.

Figure 4 shows the average Bloc Error Rate versus the number of users. $X$ axis represents the number of users and $\mathrm{Y}$ axis shows the average BLER. It is outstanding that the proposed method provides lowest BLER compared to other methods. 


\section{CONCLUSION}

This study has considered the problem of resource allocation for mobiles users in context of OFDMA system. Particularly we have used the contiguous method for subchannels construction in mobile environment. The main objective in this study is to resolve the problem of subchannels assignment to mobile users, in order to take into account the trade-off between maximization the use of resources and fairness. In this context, a new dynamic heuristic algorithm have been proposed and compared with alternatives previously proposed in the literature.

The proposed algorithm is based on statistic indicators as the average variance of subchannels gains for each mobile user and for each subchannels used by all mobile users. The assignment is performed every sub-frame delay. Numerical results show that the proposed algorithm permits to achieve a better trade-off between fairness and efficiency use of resources compared to other methods. In addition to this contribution, the new heuristic algorithm presents a low complexity and may be adopted for real-time and mobile applications, as: voice and video-call.

\section{REFERENCES}

Bayan, A.F., T.C. Wan and S. Ramadass, 2011. Delay Analysis and System Capacity Control for Mobile WiMAX Relay Networks. J. Comput. Sci. 6: 11371143. DOI: $10.3844 /$ jcssp.2010.1137.1143

Sadr, S., A. Anpalagan and K. Raahemifar, 2009. Suboptimal rate adaptive resource allocation for downlink OFDMA systems. Int. J. Vehicular Technol., 2009: 10-10. DOI: 10.1155/2009/891367

Cheong, Y.W., R.S. Cheng, K.B. Lataief and R.D. Murch, 1999. Multiuser OFDM with adaptive subcarrier, bit, and power allocation. IEEE J. Selected Areas Commun., 17: 1747-1758. DOI: 10.1109/49.793310

Hwang, S., J. Park, Y.S. Jang and H.S. Cho, 2008. A heuristic method for channel allocation and scheduling in an ofdma system. ETRI, J., 30: 741-743.

Jang, J. and K.B. Lee, 2003. Transmit power adaptation for multiuser OFDM systems. IEEE J. Selected Areas Commun., 21: 171-178. DOI: 10.1109/JSAC.2002.807348
Liejun, W., 2011. An improved water-filling power allocation method in MIMO OFDM systems. Inform. Technol. J., 10: 639-647. http://www.doaj.org/doaj?func=abstract\&id=695385

Marabissi, D., D. Tarchi, R. Fantacci and A. Biagioni, 2008. Adaptive subcarrier allocation algorithms in wireless ofdma systems. Proceedings of the International Conference on Communications, May, 19-23, IEEE Xplore Press, Beijing, pp: 34753479. DOI: $10.1109 /$ ICC.2008.653

Papoutsis, V.D., I.G. Fraimis and S.A. Kotsopoulos, 2010. A novel fairness-aware resource allocation scheme in multiuser siso-ofdma downlink. Int. J Vehicular Technol., 2010: 10-10. DOI: $10.1155 / 2010 / 432762$

Rhee, W. and J.M. Cioffi, 2000. Increase in capacity of multiuser ofdm system using dynamic subchannel allocation. Vehicular Technology Conference Proc. Vehicular Technol Conf., 2:1085-1089. DOI: 10.1109/VETECS.2000.851292

Sen, C., J. Hong and L. Xi, 2009. A novel adaptive resource allocation scheme in downlink ofdmabased system. Proceedings of the International Conference on Wireless Communications Networking and Mobile Computing, Sep. 24-26, IEEE Xplore Press, Beijing, pp: 1-4. DOI: 10.1109/WICOM.2009.5305623

Seong, K., M. Mohseni and J.M. Cioffi, 2006. Optimal resource allocation for OFDMA downlink systems. Proceeding of the Information International Symposium on Information Theory, July, 9-14, IEEE Xplore Press, Seattle, WA., pp: 1394-1398. DOI: 10.1109/ISIT.2006.262075

Shen, Z., J.G. Andrews and B.L. Evans, 2005. Adaptive resource allocation in multiuser ofdm systems with proportional rate constraints. IEEE Trans. Wireless Commun., 4: 2726-2737. DOI: 10.1109/TWC.2005.858010

$\mathrm{Yu}, \mathrm{W}$. and R. Lui, 2006. Dual methods for nonconvex spectrum optimization of multicarrier systems. IEEE Trans. Commun., 54: 1310-1322. DOI: 10.1109/TCOMM.2006.877962

Zhu, H. and J. Wang, 2010. Adaptive chunk-based allocation in multiuser OFDM systems. Proceeding of the Wireless Communications and Networking Conference, Apr. 18-21, IEEE Xplore Press, Sydney, Australia, pp: 1-6. DOI: 10.1109/WCNC.2010.5506269

Zunxiong, L., X. Zheng, F. Xingle and L. Zhaogan, 2009. Design of TDD/TDMA 4G system with link adaptation. Inform. Technol. J., 8: 770-775. 